\title{
Arbor
}

\section{Familia: Consumo y ahorro}

Manuel Navarro López

Arbor CLXXIV, 685 (Enero 2003), 99-114 pp.

Es obvio que el contenido de este trabajo deberá moverse entre la sociología de la familia y la del consumo, aunque solo sea como referentes teóricos. Sin embargo, tales vínculos deberán ser solo puntos de apoyo para analizar sin ataduras académicas una visión de los comportamientos económicos que tienen su localización primaria en la familia. Consecuentemente, tendremos que hacer una descripción de esos comportamientos y sus contenidos, su evolución y su diversificación, retomando los motivos más recurrentes al uso, pero con la orientación hacia la familia como institución y como grupo en las sociedades avanzadas actuales.

La perspectiva teórica más difundida en las ciencias sociales es que la familia o el grupo de parentesco ha sido el eje de toda vida social y económica en las sociedades más antiguas, perviviendo en gran medida esa situación hasta bien entrada la denominada sociedad industrial; en la que, como consecuencia de una especialización funcional, habrían aparecido instituciones complejas que vaciarían de contenido las actividades sociales y económicas que antes ejercía la familia. Según esto, se le habrían arrebatado progresivamente funciones como la educación, la asistencia sanitaria, la inserción laboral, las actividades productivas, el ocio activo y todas aquellas que formaban la totalidad de la vida de las personas en otros tiempos. De tal modo, que quedaría como el reducto casi único de las relaciones primarias y de la expresión de la personalidad completa de los individuos.

El enfoque sociológico de la familia se especializa en el estudio del grupo, su composición, las relaciones entre sus miembros y los valores en los que se sustentan. Consecuentemente, se estudian los 
cambios sociales que ocasionan transformaciones diversas en el interior de la familia y en la forma en que las personas establecen sus relaciones de parentesco y convivencia. En primer lugar, el proceso de democratización de las relaciones internas de la pareja, que originalmente protagoniza el movimiento de liberación de la mujer y que se extiende al resto sus miembros posteriormente. Pero, también otros como el que proviene del alargamiento de la edad media de vida o la aparición de vías alternativas de relación afectiva, parejas de hecho, hogares monoparentales, parejas homosexuales, matrimonios sucesivos, parejas sin hijos, etc. Predomina el análisis de los efectos que sobre la familia tienen otros procesos y cambios sociales, trasladando en gran medida el origen de las transformaciones al resto de la sociedad. En esa lógica se deriva a una interpretación de la información empírica sobre la familia en términos de una tendencia al debilitamiento creciente de los lazos familiares, el predominio de la sociedad de los individuos, la focalización hacia las instituciones secundarias y la centralidad de la vida profesional y del trabajo.

Junto a esta orientación cabe subrayar en los momentos actuales la revalorización del papel de la familia como institución, tanto en la imagen que de la misma tienen los individuos como por las funciones que objetivamente está desempeñando de una forma sustantiva para la sociedad. Algunas conclusiones en exceso reduccionistas, han llevado en el pasado reciente a profundizar en la familia como el territorio exclusivo de las relaciones íntimas entre las personas, poniéndola así al borde de su irrelevancia como institución social. Aparentemente, a la vista de esas observaciones, la familia sería cada vez más innecesaria desde el punto de vista funcional para la sociedad. Sin embargo, hay que contrastar esas observaciones y sus conclusiones con otros hechos que nos pondrían de relieve, perspectivas alternativas. Bajo esa óptica, la familia aparecería como una institución clave en las sociedades actuales, no solamente por el desempeño de las funciones especializadas que le atribuyen, sino por la resolución de problemas sociales y por su progresiva mayor importancia en la economía.

Por ejemplo, algunas teorías han sustentado la creencia de que la sociedad del bienestar, al satisfacer por otras vías las necesidades materiales, permite a los individuos desligarse de los lazos familiares a voluntad. Sin embargo, la sociedad del bienestar se ha quedado siempre en cotas menos confortables y la familia ha sido casi el único refugio para el individuo en situaciones límite. En este sentido, cuanto más compleja es una sociedad y más ha diversificado y especializado sus instituciones, más esencial está resultando ser el papel de la familia 
como complemento o sustitución de la ineficiencia o insuficiencia de esas instituciones. Pero, además en el interior de la familia se toman decisiones que trascienden su entorno con repercusiones en los espacios públicos, sociales, políticos y económicos y que cada vez son tenidas más en cuenta, porque conforman esos espacios como resultado de la suma de esas decisiones que las sociedades democráticas y de mercado colocan en su centro constitutivo.

En este sentido, muchas decisiones y en especial las económicas trascienden la realidad física de la familia-hogar y deben ser tenidas en cuenta las redes de parentesco, al menos en primera línea, pues se producen muchos flujos económicos entre padres e hijos independizados. No obstante, es cierto que existe una realidad empírica de personas solas, sin lazos familiares, y paralelamente el debilitamiento o la desaparición de las relaciones familiares.

Vamos, pues, a internarnos en el consumo y en el ahorro de las familias, intentando ir algo más allá de la descripción de comportamientos consumistas, trabajo ya muy trillado, para desarrollar sobre esa base la hipótesis expuesta sobre el papel de importancia creciente que está ejerciendo la familia en la sociedad y de forma concreta en la economía, si bien nos fijaremos también en los efectos que los cambios económicos ejercen sobre el grupo familiar.

\section{Consumo}

La satisfacción de las necesidades materiales de los individuos es el objetivo final de todo proceso económico. Tradicionalmente han estado muy vinculados la producción y el consumo y ambos se ejercían dentro o muy próximos a la familia. Por consiguiente, la familia o el grupo de parentesco eran claves para la supervivencia. Con el desarrollo de la sociedad industrial la separación de ambos procesos ha sido total, las actividades productivas salen del seno de la familia para localizarse en empresas y centros de trabajo colectivos, razón que promueve que algunas teorías hayan planteado la obsolescencia de la familia o su reducción a la satisfacción de necesidades no materiales. $\mathrm{Y}$ aunque en un primer momento del industrialismo ese efecto es real, conforme la economía ha evolucionado la familia ha recuperado parte de su papel económico de forma significativa. Por eso, vamos a tratar de condicionar tal aserto desde los propios procesos económicos que afectan a la familia, centrados en el consumo y en el ahorro. Con relación al primero y aunque sea de forma sintética, procedemos a exponer 
un abanico de elementos que pueden desarrollarse en torno al consumo de las familias:

De la cultura de la escasez a la cultura de la abundancia. En la mayoría de las sociedades europeas se puede seguir, aunque con distintos tempos, el recorrido hacia un consumo abundante, diverso y generalizado al conjunto de la población. En el caso de España ese proceso tiene fechas concretas que van desde mediados del siglo XX, y especialmente desde finales de los sesenta, hasta la actualidad; si bien, ya en los años setenta se comienza a hablar de consumo de masas, aunque solo fuese por mimetismo con otras sociedades. Además, tal evolución está documentada estadísticamente a través de las que se han denominado Encuestas de Presupuestos Familiares, cuya calificación nos indica el protagonismo de la familia como unidad de análisis de un fenómeno económico como el consumo, pero asimismo su creciente protagonismo real en la economía. El resultado de ambos es un consumo más abundante y una transformación del mismo, que constatamos en las diferentes encuestas que se han hecho en nuestro país desde 1957 (véase el gráfico 1), lo mismo que las realizadas en otros.

GRÁfICO 1: Índices de crecimiento del consumo 1958-2001

$(1958=100)$

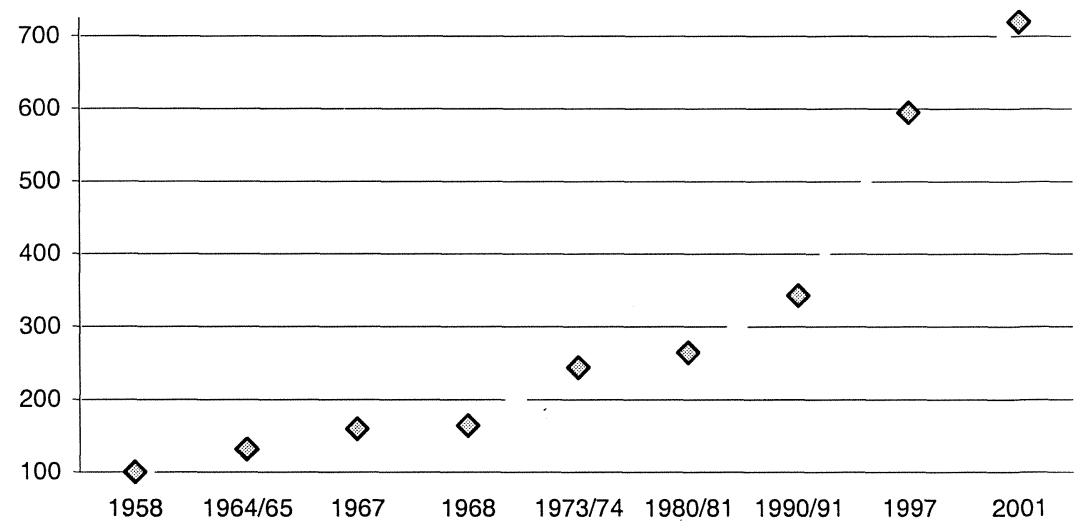

Fuente: INE, Encuestas de Presupuestos Familiares y elaboración propia ${ }^{1}$.

A lo largo de este período la sociedad española ha pasado de ser una sociedad predominantemente rural y con una economía preindustrial y, por ende, básicamente agrícola, a ser una economía de mercado 


\section{Familia: Consumo y ahorro}

moderna, con una fuerte industrialización; y paralelamente se ha ido abriendo al intercambio con otras economías y mercados. Como consecuencia, se han multiplicado el número de objetos producidos y disponibles, objetos estandarizados y homogéneos, lo que ha dado lugar a un mercado rico y diversificado, que a la vez es un mercado de masas. Mientras que en épocas anteriores, la alimentación, el vestido y los artículos más elementales de la casa y del trabajo centran toda la actividad consumista de las familias, en la actualidad esas parcelas representan una mínima parte del gasto total. Además, los bienes que cubrían esas necesidades antiguamente eran escasos y se entendía que algunos deberían durar toda la vida.

El efecto taxonómico derivado de las categorías de codificación de las EPF consagra unos grupos de gastos que dirigen la representación que tenemos de lo que es el consumo en nuestras sociedades en una progresión que va de los gastos vinculados a supuestas necesidades básicas hasta los que satisfacen necesidades prescindibles: alimentación, vestido, vivienda, enseres, transportes, salud, educación, cultura, actividades de ocio, turismo, servicios personales, seguros y similares. La evolución de los capítulos de gasto más agregados, definidos desde la Encuesta de Cuentas Familiares, de 1957, se recoge en el gráfico 2.

GRÁFICO 2: Distribución proporcional de los gastos de consumo, 1958-1999

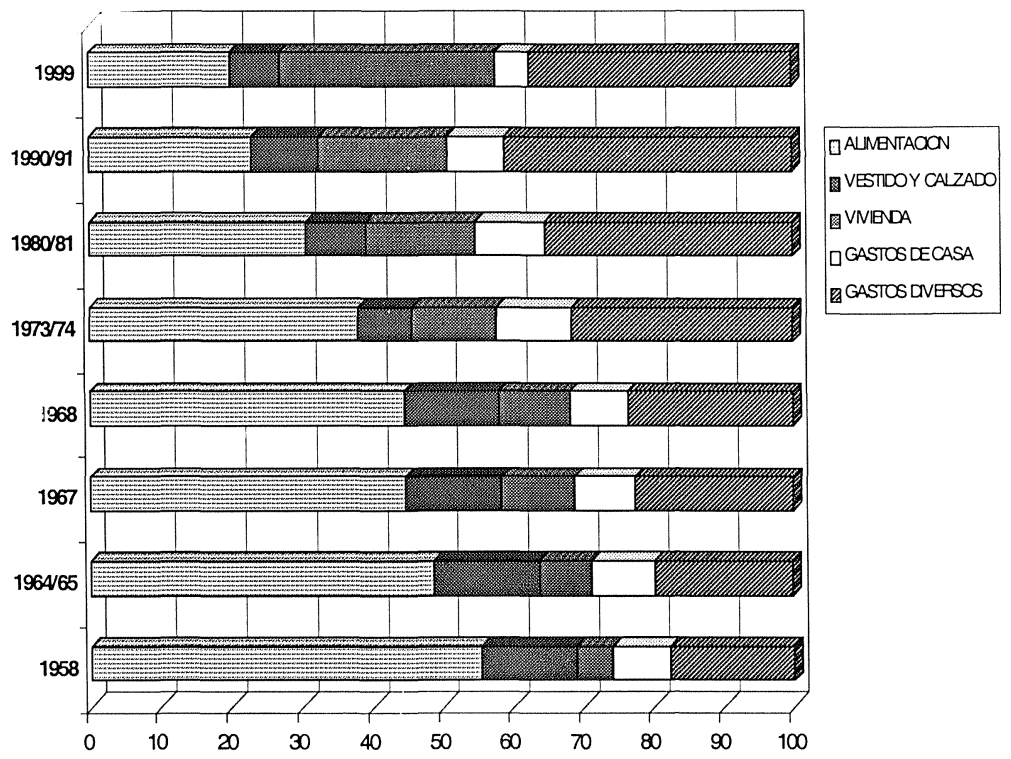

Fuente: INE, Encuesta de Presupuestos Familiares ${ }^{2}$. 
En la actualidad la mayor disponibilidad de recursos económicos posibilita que aparezcan nuevos consumos, sin que por ello se degraden los anteriores. Por el contrario, éstos quedan intensificados. Así, aunque los gastos de alimentación son ahora menos gravosos para la economía familiar, sin embargo, son mayores en términos absolutos. La tendencia fundamental de las pautas alimenticias de los españoles ha estado dirigida hacia una nutrición más rica y diversificada, pero también más industrializada, habiendo dado lugar a importantes modificaciones de los gustos y hábitos dietéticos de los españoles ${ }^{3}$. La dieta media no sólo se ha diversificado sino que se ha enriquecido en valor nutritivo: frente a una situación de escasez se ha pasado a otra de abundancia, la cual, aunque en algún caso haya podido tener un resultado poco adecuado para la salud, (por ejemplo por el exceso de calorías); en general ha sido decisiva en el alargamiento de la esperanza media de vida.

Algo similar, si bien de forma más lenta y menos radical, ha sucedido con la vivienda. De una situación de escasez generalizada, e incluso de hacinamiento para gran parte de la población urbana, se ha pasado a una satisfacción amplia de las demandas de los españoles, hasta el punto de que la mayor parte de la población dispone en la actualidad de una habitación propia. A ello se une el ato nivel de equipamiento del hogar, tal como refleja el gráfico 3 , en el que se recogen solamente algunos significativos.

GRÁFICO 3: Equipamientos de los hogares (en porcentajes)

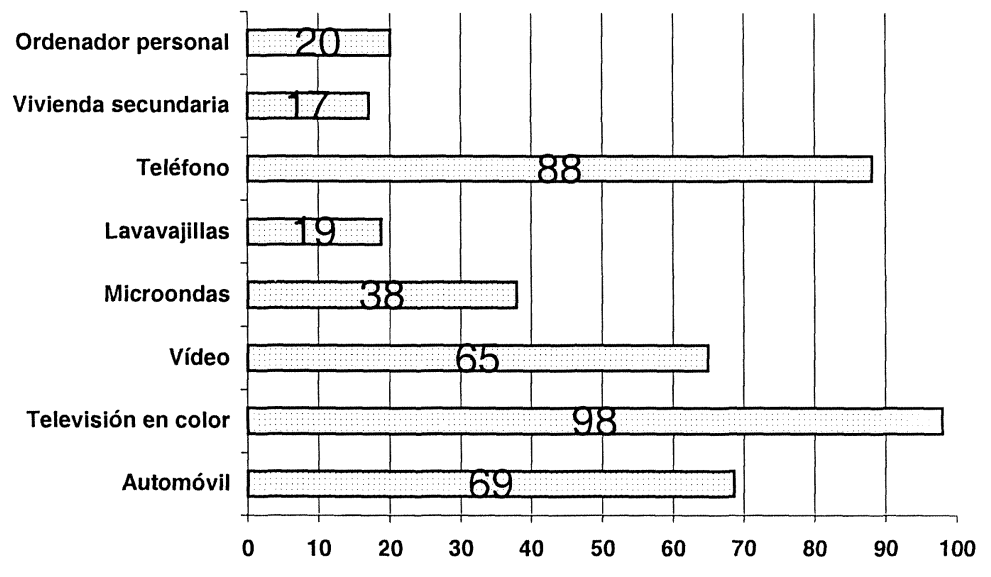

FUENTE: INE, Panel de Hogares, 1996. 
Por otra parte, han surgido nuevos grupos de consumos como los relativos a cultura y enseñanza, transportes y comunicaciones, cuidados personales, esparcimiento y ocio, y viajes y turismo, entre otros muchos, con un peso cada vez mayor en la estructura global del consumo, tal como hemos visto anteriormente.

Estilos y modos de vida. Todos los cambios experimentados por la capacidad consumidora, que suponen cambios en el modo de vida, han afectado a la familia en múltiples aspectos, de entre los cuales podríamos resaltar:

- El incremento del consumo y del equipamiento ha hecho la vida dentro del hogar más confortable y ha incorporado más elementos de ocio, con lo cual los miembros de la familia han tendido a pasar más tiempo dentro de la casa, favoreciendo sus relaciones mutuas.

- La individualización de una parte creciente de los gastos familiares (comidas fuera de casa, vestido, transportes, ocio, etc.), emancipa y da cierta autonomía a todos los miembros de la familia y contribuye a la democratización de sus relaciones, con una mayor libertad en su convivencia.

- El equipamiento del hogar y la industrialización de la alimentación han simplificado el trabajo doméstico, lo que ha sido un punto de apoyo esencial para la incorporación de la mujer al trabajo fuera del hogar.

- El alargamiento de la esperanza de vida como consecuencia de la mejora de las condiciones materiales y especialmente de la alimentación y los cuidados médicos, dilata el ciclo de vida y crea nuevas formas de hogar.

- Una mayor dedicación al consumo, conforme éste es más cuantioso y variado, con el desplazamiento de muchas actividades familiares a los centros comerciales.

- Una mayor libertad de elección entre diferentes tipos de consumo, dando lugar a una diversificación de los estilos de vida y de los comportamientos consumistas de las familias.

La familia ha ido acomodándose a estos y otros cambios similares sin perder por ello su relevancia y su identidad como grupo, pero incrementando su importancia en la vida social. No obstante, los efectos no han sido iguales para todos ni las posibilidades de acceso al consumo. Se ha producido una diversificación de los consumos, pero también han emergido desigualdades sociales. 
La teoría de la sociedad de consumo de masas nos ha legado el discurso sobre el crecimiento del gasto de los hogares, de la homogeneización de los consumos básicos como resultado de la industrialización y la difusión de una gran clase media, definida desde la cultura del consumo, por su uniformidad en el modo de vida. La producción artesanal es por sí misma exclusivista y diferenciadora, y se corresponde con el modelo de consumo y, en definitiva de vida, de la sociedad estamental. La sociedad industrial perpetúa las diferencias a lo largo del siglo XIX, con tres modos básicos de vida urbana y de consumo excluyentes: el de la burguesía, el de la pequeña burguesía y el de la clase obrera. Esta diferenciación se prolonga hasta el desarrollo de las industrias de consumo y la expansión de una clase media socialmente homogénea. La producción industrial de bienes de consumo es igualadora en la funcionalidad y en la forma del producto, aunque perpetua las desigualdades en la cantidad y en la calidad de lo que se puede consumir. Pero, es en sí misma democratizadora, permitiendo el acceso de todos al consumo de cualquier objeto, que es igual para todos los consumidores. La sociedad de consumo de masas resume es ese término el proceso de igualación que se ha operado.

El resultado es la aparición de múltiples y diferentes estilos de vidas que tienen su fundamento en las opciones de consumo hechas posibles por la abundancia: por ejemplo, privilegiando el ocio, la alimentación, la moda en el vestido, la educación, etc. Se establecen diferenciaciones sociales de todo tipo, pero también distinciones sociales, sustentadas en la capacidad económica y en el acceso a bienes y servicios exclusivos, que se reproducen según el crecimiento económico permite su difusión masiva. Esas diferencias y distinciones se entrecruzan con las variaciones en las formas familiares y en los grupos de edad, con un balance final de heterogeneidad en los modelos de vida, a las que se van a sumar las diferencias culturales provenientes de la inmigración.

Hay muchos otros aspectos a considerar en los estilos de vida de las familias, pero los más fundamentales giran o han girado en torno a dos dimensiones, la seguridad y planificación del futuro y el consumismo que induce la abundancia. Así, podemos señalar los siguientes:

- Frente a la lógica de la incertidumbre que ha presidido la economía familiar en otros tiempos, motivada por la recurrencia de catástrofes naturales, epidemias, guerras y otras; o bien la incidencia de crisis económicas incontroladas, en épocas más recientes, en la actualidad las familias han asumido la lógica 
de la seguridad y viven el futuro con expectativas de continuidad en el consumo y en el bienestar económico.

- Se planifican cada vez más las etapas del ciclo vital, con la confianza de llegar a las más avanzadas y mantener el nivel de vida. Ejemplo de ello es el desarrollo de los seguros, los planes de pensiones y otras formas de ahorro y la inversión en educación para garantizar el futuro de los hijos.

- También la planificación en el tiempo de las prácticas consumistas, por ejemplo con las compras a plazos.

- La asimilación del cálculo económico racional, por ejemplo, comparando precios y calidades.

- Generalización del consumismo en diferentes direcciones: el despilfarro, la obsolescencia planificada de los bienes duraderos y la cultura del usar y tirar.

- Difusión de la moda en cada vez más objetos de consumo.

- Facilidad para cambiar el modelo de consumo hacia otros más sofisticados, con una adaptación rápida a las innovaciones tecnológicas.

- El consumo ostentoso y simbólico de la posición social o de los modelos de vida difundidos por los medios de comunicación.

La sociologización de la economía. Probablemente el aspecto menos considerado acerca de la influencia de la familia en las sociedades avanzadas sea el del papel económico que desempeña, hasta el punto de que las familias como grupos organizados se han convertido en una pieza clave del sistema socioeconómico actual. Anteriormente, hemos manejado la perspectiva de cómo los cambios en la economía han inducido otros en las familias y en sus estilos de vida, ahora el enfoque es el contrarío: cómo la estructura familiar y las decisiones que se toman en su interior repercuten en el conjunto de la economía. La sociología de la familia no parece haber considerado este fenómeno hasta el presente, salvo quizás de forma tangencial; no obstante, la economía sí está incorporando progresivamente este análisis.

Como hemos explicado antes, el advenimiento de la economía industrial desplazó el papel económico de la familia hacia las unidades productivas especializadas; consecuentemente, la ciencia de la economía se encaminó al estudio de las empresas, el comercio, las instituciones financieras y los grupos económicos de mayor peso, en consonancia con el desarrollo de esos sectores. La economía de la familia perdió importancia e interés como objeto de estudio, pues, siendo una resultante del resto de fuerzas económicas, su destino final, a lo sumo, y por tanto su papel era residual y pasivo. 
Pero, en la actualidad las economías familiares han alcanzado progresivamente una función económica que se puede calificar de estratégica y que tiene básicamente dos aspectos fundamentales, el consumo y el ahorro, junto a otros menos estudiados y que pueden denominarse redistributivos. En el primer caso, las familias se convierten en centros de consumo complejos, sofisticados y decisivos en términos «micro» y «macroeconómicos», con una entidad como no había tenido nunca en toda la historia, hasta el punto de calificar a toda la sociedad como «sociedad de consumo». Las decisiones de compra que se toman en el ámbito familiar tienen progresivamente una repercusión primordial para la producción económica y para el equilibrio y crecimiento de la economía global. La importancia de estos gastos no se cifra solamente en su trascendencia económica, sino también en su «discrecionalidad». Como están menos constreñidos a necesidades de pura supervivencia que los anteriores, las situaciones y condiciones de vida de los consumidores o sus preferencias personales o familiares comienzan a hacer aparición, introduciendo elementos de variación que conduce a una cierta diversificación de los modelos de consumo y a una relativa libertad de elección, que se concreta en múltiples conductas consumistas, determinadas por gustos personales y modas o situaciones familiares, de edad, lugar de residencia, posición social, etcétera.

En el plano de la microeconomía las decisiones de consumo de las familias son relativamente concluyentes para el éxito de una nueva tecnología, la introducción de un nuevo producto, de una marca e, incluso, la supervivencia de una empresa. Conforme las industrias de consumo, de comercio minorista y los servicios han proliferado, más se supeditan a las necesidades, gustos o deseos de los consumidores. Y según ha ido aumentando el poder adquisitivo de éstos, un mayor número de actividades y empresas económicas dependen de esas demandas. En este sentido, han venido siendo objeto de interés creciente para los empresarios, los cuales han implementado dos instrumentos imprescindibles hoy día para la mayoría de las empresas: por una parte, los estudios de mercado, con objeto de conocer la demanda, es decir, los gustos de las familias tamizados por su poder de compra; $\mathrm{y}$, por otra, la publicidad, cuyo fin es crear esos gustos o la imagen del producto que los satisfará ${ }^{4}$.

En el ámbito de la macroeconomía, las familias, en tanto que economías domésticas según la metodología de las contabilidades nacionales, son también una pieza relevante de las economías actuales; pues, como señaló en su momento G. Katona ${ }^{5}$, el consumo familiar no solamente es mayor cuantitativamente, sino que se hace discrecional, 
de tal forma que las decisiones de millones de consumidores pueden ser determinantes para la demanda global, en la medida de que se trata de una demanda agregada, suma de miles o millones de demandas familiares, que pueden verse afectadas por las diversas opciones que se tomen en cada momento, por ejemplo entre ahorro y consumo, o entre vivienda o vacaciones. Es de este modo como las preferencias de las familias pueden interesar variables económicas como el índice de precios al consumo o desequilibrios productivos según las variaciones en la composición de los gastos familiares. Esto está llevando a una preocupación creciente por los estudios sociológicos de las expectativas y valoraciones de la economía que tienen los consumidores, con la incorporación de indicadores del consumo familiar, como el índice de sentimiento del consumidor y sus variantes, a la panoplia de instrumentos de análisis de la economía.

\begin{abstract}
Ahorro
Conforme el nivel de renta ha ido aumentando las familias han podido permitirse no solamente un consumo más diversificado y cuantioso sino que progresivamente han podido realizar un ahorro también más controlado y diversificado. Es cierto que en el pasado el ahorro no solo dependía de su posibilidad material, es decir de la renta disponible, sino de hábitos y estilos de vida. En España ha habido una tradición de ahorro, incluso en la pobreza, que se ha manifestado con mayor intensidad en determinadas regiones y sectores sociales. Así, por ejemplo, el campesinado ha tenido una mayor propensión cultural al ahorro que el proletariado, derivada de la situación de mayor riesgo en la que se encontraba frente a acontecimientos catastróficos incontrolables; y, a la vez, la valoración de la propiedad rústica y de su vivienda. Ese sentimiento se generaliza y arraiga de forma el ahorro se canaliza, en épocas más recientes, a la compra de la vivienda urbana, de modo preferente.

Podríamos trazar, en este contexto, una trayectoria elemental de las formas de ahorro que se corresponden con los recursos financieros que han ahorro en forma de joyas (oro) o de dinero guardado debajo del ladrillo o del colchón. En los años sesenta aparecen algunos instrumentos financieros de mayor proyección social, de los cuales la estrella es la cartilla de ahorro, estado a disposición de las familias. Así, tradicionalmente, ha primado el orientada a prevenir necesidades
\end{abstract}


extraordinarias, pero emerge ya la primera forma de inversión, la inversión en ladrillos, que se materializa en la compra de la vivienda principal y para los sectores sociales más elevados, la segunda vivienda.

A finales de los años setenta y durante los ochenta se difunde una forma más refinada de canalización del ahorro, en consonancia con las necesidades del sector público, la deuda pública y las letras del tesoro. Se inicia así un ahorro activo, buscando rentabilidades comparativas, que se amplía con otros activos financieros similares. En los noventa, se manifiesta ya una clara eclosión de la inversión activa, concretada en la Bolsa, los fondos de inversión, los planes de pensiones $\mathrm{y}$ otras fórmulas equivalentes.

Un estudio reciente de la Bolsa de Madrid ${ }^{6}$ da cuenta de estas aseveraciones, tal como recoge el gráfico 4, sobre la distribución de la inversión de las familias españolas en el año 2000, donde se puede apreciar la importancia adquirida por la compra de acciones, que se estima alcanzaría un volumen de más de 67 billones de pesetas; todo lo cual fluctuaría lógicamente con el ciclo económico y las cotizaciones.

GRÁFICO 4: Distribución porcentual de la inversión de las familias españolas

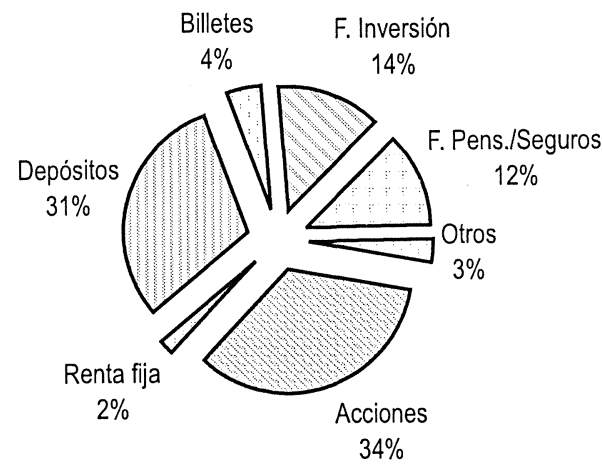

Fuente: Bolsa de Madrid, 2000

En la actualidad, hay un porcentaje importante de familias que ahorran de forma sistemática y esa proporción es aún mayor en los países más avanzados de nuestro entorno, si bien parecen existir algunas diferencias culturales, tal como expresan los datos del cuadro 1. 


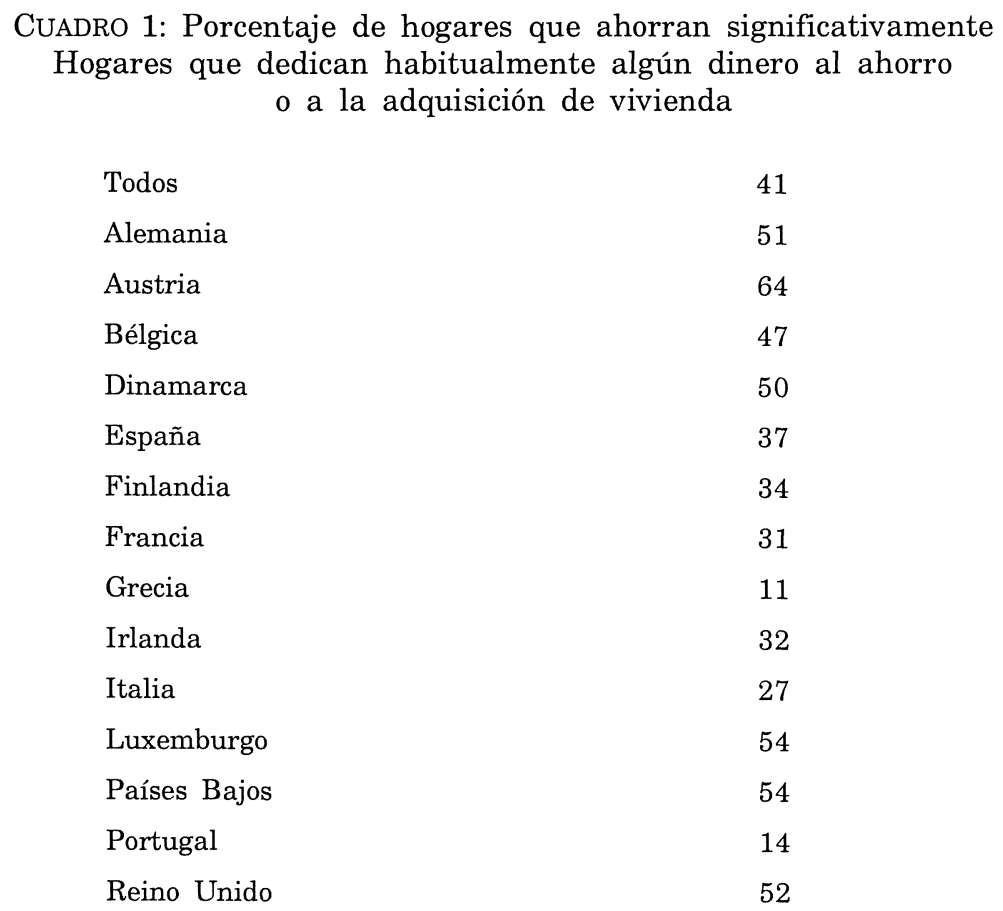

Fuente: Panel de Hogares de la Unión Europea, Resultados del tercer ciclo. Año 1996.

¿Cómo afecta o va a afectar a la familia esa disponibilidad creciente de capacidad de ahorro? Hay tres dimensiones principales:

- La vivienda, generalizada para casi todas las familias con espacio y equipamientos cada vez más confortables.

- Planificación de la vida laboral y de la vejez, con la garantía de asegurarse un nivel de vida y/o adelantar la jubilación.

- Adquisición de una cultura económica: conocimiento de la lógica de la economía, búsqueda de información, intervención activa.

Sociologización del mundo financiero. Como consecuencia de lo anterior y especialmente de la última variable, nos encontramos que las familias de fuerte poder adquisitivo, cuyo número es creciente en las sociedades más avanzadas, están adquiriendo un protagonismo especial en el campo financiero, por su capacidad de generación de ahorro, con sus repercusiones correspondientes sobre la estrategia de la inversión económica y a la postre de todo el sistema productivo. Asistimos, de este modo, a la aparición de dos fenómenos nuevos e interrelacionados. Primero, el surgimiento de decisiones de inversión grupales o condicionadas por el grupo familiar, que involucran búsqueda 
de información y despliegue de una cultura económica y financiera. Segundo, el de la envergadura económica alcanzada por las familias, en términos contables, en virtud de sus propiedades y de su posibilidad de generación de recursos económicos.

De esta forma, percibimos que las familias han dejado de ser un objetivo secundario de las instituciones financieras como clientes cautivos y homogéneos: donde antes los grandes clientes de los bancos eran las empresas, ahora son también muchas familias. De ahí que, su capacidad de generar ahorro y su libertad para definir el destino del mismo las haya convertido una pieza estratégica de la economía y del marketing financiero: cuentas nómina, tarjetas de crédito, préstamos al consumo, hipotecas, fondos de inversión, planes de pensiones y un innumerable conjunto de productos que son hoy día una parte importante de los negocios bancarios y del sector financiero.

Baste señalar que los movimientos de la Bolsas ${ }^{7}$ están dependiendo cada vez más de las decisiones de millones de individuos y familias (por lo que respecta a la sociedad española y según un reciente estudio de la Bolsa de Madrid, las familias poseen más del $40 \%$ de la capitalización bursátil, siendo de tan sólo de alrededor del $22 \%$ en los comienzos de los años noventa) ${ }^{8}$.

Es decir, hay un crecimiento importante de la capacidad financiera de las familias y esa capacidad se ejerce directamente en los mercados financieros. No del modo pasivo que ha caracterizado tradicionalmente al ahorro familiar, centrado en depósitos bancarios o renta fija, sino de un modo cada vez más activo e informado $y$, por consiguiente, con capacidad de decisión y de influir en el conjunto de los mercados y convertirse de esta manera en sujetos activos de la economía más dinámica, incluida la que se ha denominado «nueva economía».

\section{Cuestiones complementarias}

Tal como hemos anunciado anteriormente aún podríamos señalar otros aspectos, bajo otras perspectivas menos formalizadas en las ciencias sociales, que ponen de relieve la centralidad del grupo familiar en nuestras sociedades, en tanto juegan un substancial papel económico, aunque tan solo vamos a formular muy someramente algunos que constituyen, en todo caso, líneas de investigación abiertas o por abrir.

- En primer lugar, se encontraría la valoración económica de la producción realizada en el hogar o del trabajo doméstico, dónde 


\section{Familia: Consumo y ahorro}

se pueden cuantificar algunas de las funciones sociales de la familia enunciadas anteriormente ${ }^{9}$.

- En segundo lugar, también desde el punto de vista productivo, el papel emprendedor de algunos grupos familiares continuado a través del tiempo y que se resume en el concepto acuñado de «empresa familiar», si bien más desde una perspectiva sociológica que bajo los recovecos de problemas, falsos problemas, ventajas y otras gabelas que esconde la perspectiva económica.

- En tercer lugar, la función de redistribución económica inversa que se hace desde las familias hacia otros sectores en términos de trasvase de flujos monetarios, de las que citaremos a título de ejemplo, las que conciernen a impuestos, precios diferenciales en servicios regulados como electricidad, agua o gas; tasas de servicios públicos, asunción de riesgos laborales, formación profesional y otras cuya explicación rebasa aquí y ahora el propósito de este artículo.

- En cuarto lugar, la subsistencia de la familia como centro de producción en muchos países menos desarrollados, con tradición agrícola o artesanal, y como sujeto de la economía informal en los más avanzados.

Finalmente, cabría hacer un ejercicio complementario para avanzar las tendencias más importantes que pueden presidir la evolución del consumo y del ahorro en las familias, que sería tema asimismo de una investigación específica, que desbordaría los límites de este artículo ${ }^{10}$.

\section{Notas}

1 Los datos referentes a 1997 y 2001 son estimaciones en base a otros datos del INE que pueden tener un componente de error significativo para su valor exacto, pero no para la expresión gráfica del crecimiento del consumo.

2 La comparabilidad más problemática es la de los datos de 1999, por provenir de la Encuesta Continua de presupuestos y recoger un cambio metodológico en la imputación de los gastos de vivienda.

3 Menos cantidades de legumbres, pan, patatas, pastas, huevos y, sobre todo, grasas; y más de carne, pescado, verduras y hortalizas, frutas, leche y derivados, azúcar y, de modo especial, las bebidas no alcohólicas.

4 En ambos casos nos encontramos con un planteamiento eminentemente sociológico, bien sea de investigación social o de psicología social. Pero, en ambos los sociólogos han perdido su protagonismo a favor de economistas y publicistas.

5 Aquí Katona no hizo más que "sociologizar" los planteamientos keynesianos y retomar una larga tradición de sociólogos y economistas empíricos que desde el siglo XIX venían estudiando la evolución del consumo y los niveles de vida de las familias, 
tradición desconocida al parecer por algunos practicantes actuales del marketing metidos a sociólogos.

5 Citado de Expansión, 15-12-2000, p. 21.

6 El número y, en menor medida, el volumen de las transacciones, pero no el control de la Bolsa y mucho menos de las empresas. Por eso, es impropio o sarcástico, según se quiera, hablar de capitalismo popular.

7 Según un «Estudio sobre el Ahorro y los Mercados Financieros en España», citado en Expansión, 5 de Julio de 2000. Las acciones mantenidas por inversores individuales sería del $30 \%$, por delante de países como el Reino Unido o Alemania. Otra cosa es la distribución de esa riqueza entre las familias españolas. A ambos fenómenos no es ajena la importancia de la empresa familiar a la que nos referimos más adelante.

8 Esta línea ha sido emprendida por algunos autores para llamar la atención especialmente sobre el trabajo de la ama de casa. Por ejemplo, M $\mathrm{M}^{\mathrm{a}}$ Ángeles Durán, "producción doméstica», en Salustiano del Campo (ed.), Tendencias Sociales en España (1960-1980), Fundación BBV, Bilbao, 1993, vol. III.

9 Me remito a una aproximación a esta cuestión en el trabajo M. Navarro, «Tendencias de Desigualdad en el Consumo", en J. F. Tezanos, y R. Sánches Morales (eds.), Tendencias de Futuro en la Sociedad Española, Ed. Sistema, Madrid, 1998, pp. 375-399. 